\title{
THE FERMION SIGN PROBLEM AND HIGH DENSITY EFFECTIVE THEORY
}

\author{
D. K. HONG* \\ Department of Physics, \\ Pusan National University, \\ Pusan 609-735, Korea \\ E-mail:dkhong@pusan.ac.kr \\ S. D. H. HSU ${ }^{\dagger}$ \\ Institute of Theoretical Science \\ University of Oregon, \\ Eugene, OR 97403-5203 \\ E-mail: hsu@duende.uoregon.edu
}

\begin{abstract}
We investigate the positivity of the Euclidean path integral measure for low-energy modes in dense fermionic matter. We show that the sign problem usually associated with fermions is absent if one considers only low-energy degrees of freedom. We describe a method for simulating dense QCD on the lattice and give a proof using rigorous inequalities that the color-flavor locked $(\mathrm{CFL})$ phase is the true vacuum of three flavor, massless QCD.
\end{abstract}

\section{Introduction}

Euclidean quantum chromodynamics (QCD) with a non-zero chemical potential has a complex measure, which has made lattice simulation particularly difficult ${ }^{1}$. (Lattice simulation of the QCD phase boundary at finite density $^{2,3}$ has been a topic of recent interest.) This problem is often referred to as the sign problem, because, by appropriately grouping terms, quantities such as the partition function can be written as a sum over real, but potentially negative, terms. (That this grouping can be accomplished

\footnotetext{
*Work partially supported by the academic research fund of the Ministry of Education, Republic of Korea, Project KRF-2000-015-DP0069, and the USA-Korea Cooperative Science Program, NSF 9982164.

†Work partially supported by the U.S. Deptartment of Energy, DE-FG06-85ER40224 and the USA-Korea Cooperative Science Program, NSF 9982164.
} 
is in many systems a consequence of a discrete symmetry such as parity or time-reversal invariance.) Indeterminate signs are enough to preclude use of importance sampling, the main technique for speeding up Monte Carlo integration. It is important to note that while the sign problem often arises in systems of fermions, it is neither inevitable nor inescapable. For example, in QCD at zero chemical potential and in the Hubbard model at half filling one can organize the sum so that terms are real and positive.

Analytical work in color superconductivity ${ }^{4}$ has demonstrated a rich phase structure at high density, and stimulated interest in QCD at nonzero baryon density. Several experiments have been proposed to probe matter at density of a few times nuclear matter density ${ }^{5}$. Even rudimentary information about the behavior of dense matter would be useful to the experimental program, as well as to the study of compact astrophysical objects such as neutron stars. Recently ${ }^{6}$, we showed that QCD near a Fermi surface has positive, semi-definite measure. In the limit of low energies, the contribution of the remaining modes far from the Fermi surface can be systematically expanded, using a high density effective theory previously introduced by one of us ${ }^{7,8}$. This effective theory is sufficient to study phenomena like color superconductivity, although quantities like the equation of state may be largely determined by dynamics deep in the Fermi sea.

The expansion about the Fermi surface is in powers of $1 / \mu$, where $\mu$ is the chemical potential. For this expansion to be controlled, the ultraviolet cutoff of our effective theory must be less than $\mu$, or equivalently the scale of the physics of interest must be small relative to the chemical potential. In QCD at asymptotic density, the superconducting gap is exponentially small, so this condition is satisfied. However, it is also quite possible that at intermediate densities (e.g., those inside a neutron star) the gap is somewhat smaller than $\mu$, providing us with an additional small dimensionless parameter. Even if this is not the case, the power expansion of the effective theory is qualitatively different from the usual perturbation in $\alpha_{s}$, and therefore worth exploring.

\section{Example: $(1+1)$ Dimensions and Beyond}

We begin with an example that illustrates the basic ideas in a simple setting. Consider the Euclidean (1+1) action of non-relativistic fermions interacting with a gauge field $\mathrm{A}$

$$
S=\int d \tau d x \psi_{\sigma}^{*}\left[\left(-\partial_{\tau}+i \phi+\epsilon_{F}\right)-\epsilon\left(-i \partial_{x}+A\right)\right] \psi_{\sigma}
$$


where $\epsilon(p)$ is the energy as a function of momentum (e.g. $\left.\epsilon(p) \approx \frac{p^{2}}{2 m}+\cdots\right)$. In (1) and below we may consider it as a function of the operator $\left(-i \partial_{x}+A\right)$. The dispersion relation in the presence of the chemical potential $\epsilon_{F}$ is: $E(p)=\epsilon(p)-\epsilon_{F}$, and a low energy mode must have momentum close to $\pm p_{F}$, where $\epsilon\left( \pm p_{F}\right)=\epsilon_{F}$. The Fermi surface in $(1+1)$ dimensions is reduced to the two points $p= \pm p_{F}$. Near these points we have

$$
E\left(p \pm p_{F}\right) \approx \pm v_{F} p
$$

where $v_{F}=\partial E /\left.\partial p\right|_{p_{F}}$ is the Fermi velocity.

The action (1) is not obviously positive. In fact, the operator in brackets [ ... ] clearly has Hermitian as well as anti-Hermitian components, and hence complex eigenvalues.

Let us assume that the gauge field has small amplitude and is slowly varying relative to the scale $p_{F}$. We will extract the slowly varying component of the fermion field to construct a low energy effective theory involving quasiparticles and gauge fields. This effective theory will have positive, semi-definite determinant.

First, we extract the quasiparticle modes (we suppress the spin index in what follows)

$$
\psi(x, \tau)=\psi_{L} e^{+i p_{F} x}+\psi_{R} e^{-i p_{F} x},
$$

where the functions $\psi_{L, R}$ are slowly varying. To simplify the action, we use the identity

$$
e^{ \pm i p_{F} x} E\left(-i \partial_{x}+A\right) e^{\mp i p_{F} x} \psi(x) \approx \pm v_{F}\left(-i \partial_{x}+A\right) \psi(x)
$$

to obtain ${ }^{\mathrm{a}}$

$S_{\mathrm{eff}}=\int d \tau d x\left[\psi_{L}^{\dagger}\left(-\partial_{\tau}+i \phi+i \partial_{x}-A\right) \psi_{L}+\psi_{R}^{*}\left(-\partial_{\tau}+i \phi-i \partial_{x}+A\right) \psi_{R}\right]$

We can write this in a more familiar form by introducing the Euclidean $(1+1)$ gamma matrices $\gamma_{0,1,2}$, which are Hermitian and can be taken as $\gamma_{i}=\sigma_{i}$ where $\vec{\sigma}$ are the Pauli matrices. Using $\psi_{L, R}=\frac{1}{2}\left(1 \pm \gamma_{2}\right) \psi$ we obtain

$$
S_{\mathrm{eff}}=\int d \tau d x \bar{\psi} \gamma^{\mu}\left(\partial_{\mu}+i A_{\mu}\right) \psi \equiv \int d \tau d x \bar{\psi} \not D \psi
$$

a For simplicity we set $v_{F}=1$. Alternatively it could be absorbed in the definition of the spatial $\gamma_{1}$ as in QCD below. 
Since the gamma matrices are Hermitian, and the operator $\left(\partial_{\mu}+i A_{\mu}\right)$ is anti-Hermitian, the operator $\not D$ in (6) has purely imaginary eigenvalues. However, because $\gamma_{2}$ anticommutes with $\not D$, the eigenvalues come in conjugate pairs: given $\not D \phi=\lambda \phi$, we have

$$
\not D\left(\gamma_{2} \phi\right)=-\gamma_{2} \not D \phi=-\gamma_{2} \lambda \phi=-\lambda\left(\gamma_{2} \phi_{n}\right) .
$$

Hence the determinant $\operatorname{det} \not D=\prod \lambda^{*} \lambda$ is real and positive semi-definite.

Thus, by considering only the low-energy modes near the Fermi points of the original model (1), we obtain an effective theory with desirable positivity properties. Note that it is necessary that the interactions (in this case, the background gauge field A) not couple strongly the low-energy modes to fast modes which are far from the Fermi points. This is a reasonable approximation in many physical situations, where it is the interactions among quasiparticles that are of primary interest. In what follows, we will apply this basic idea to more complex models such as QCD.

It is straightforward to go beyond $(1+1)$ dimensions. Consider an electron system, described by

$$
L=\psi^{\dagger}\left[i \partial_{t}-\epsilon(\vec{p})\right] \psi+\mu \psi^{\dagger} \psi
$$

where $\epsilon(\vec{p})$ is the electron energy, a function of momentum $\vec{p}$. It is interesting to note that the non-relativistic system already has a sign problem even at the zero density, $\mu=0$, though the free case does not suffer this, thanks to the separation of variables. In fact, it is quite unusual to have a system like vacuum QCD which has no sign problem. In Euclidean space the electron determinant is

$$
M=-\partial_{\tau}-\epsilon(\vec{p})+\mu .
$$

The first term in operator (8) is anti-Hermitian, while the rest are Hermitian. Since there is no constant matrix $P$ in the spin space that satisfies $M^{\dagger}=P M P^{-1}$, it has a sign problem in general.

Let us decompose the fermion momentum as

$$
\vec{p}=\vec{p}_{F}+\vec{l} \text {. }
$$

Again, the Fermi momentum is defined to be a momentum at which the energy equals to the chemical potential at zero temperature: $\mu=\epsilon\left(p_{F}\right)$, and the Fermi velocity is defined as

$$
\vec{v}_{F}=\left.\frac{\partial \epsilon(p)}{\partial \vec{p}}\right|_{p=p_{F}} .
$$


If we are interested in low energies, $|\vec{l}| \ll p_{F}$, we may integrate out the fast modes to get an effective operator,

$$
M_{\mathrm{EFT}}=-\partial_{\tau}-\vec{v}_{F} \cdot \vec{l},
$$

which has complex eigenvalues. However, when we include the $-\vec{v}_{F}$ sector, we have $M_{\mathrm{EFT}}\left(\vec{v}_{F}\right) M_{\mathrm{EFT}}\left(-\vec{v}_{F}\right) \leq 0$ (i.e., has real negative eigenvalues), assuming $\epsilon(\vec{p})=\epsilon(-\vec{p})$. We again see that the sign problem in the electron system is alleviated in the low-energy effective theory.

\section{QCD}

Let us recall why the measure of dense QCD is complex in Euclidean space. We use the following analytic continuation of the Dirac Lagrangian to Euclidean space:

$$
x_{0} \rightarrow-i x_{E}^{4}, \quad x_{i} \rightarrow x_{E}^{i} ; \gamma_{0} \rightarrow \gamma_{E}^{4}, \quad \gamma_{i} \rightarrow i \gamma_{E}^{i} \quad .
$$

The Euclidean gamma matrices satisfy

$$
\gamma_{E}^{\mu \dagger}=\gamma_{E}^{\mu}, \quad\left\{\gamma_{E}^{\mu}, \gamma_{E}^{\nu}\right\}=2 \delta^{\mu \nu} .
$$

The Dirac-conjugated field, $\bar{\psi}=\psi^{\dagger} \gamma^{0}$, is mapped into a field, still denoted as $\bar{\psi}$, which is independent of $\psi$ and transforms as $\psi^{\dagger}$ under $S O(4)$. Then, the grand canonical partition function for QCD is

$$
Z(\mu)=\int \mathrm{d} A_{\mu} \operatorname{det}(M) e^{-S\left(A_{\mu}\right)},
$$

where $S\left(A_{\mu}\right)$ is the positive semi-definite gauge action, and the Dirac operator

$$
M=\gamma_{E}^{\mu} D_{E}^{\mu}+\mu \gamma_{E}^{4},
$$

where $D_{E}=\partial_{E}+i A_{E}$ is the analytic continuation of the covariant derivative. The Hermitian conjugate of the Dirac operator is

$$
M^{\dagger}=-\gamma_{E}^{\mu} D_{E}^{\mu}+\mu \gamma_{E}^{4}
$$

The first term in (15) is anti-Hermitian, while the second is Hermitian, hence the generally complex eigenvalues. When $\mu=0$, the eigenvalues are purely imaginary, but come in conjugate pairs $\left(\lambda, \lambda^{*}\right)$ b, so the resulting determinant is real and positive semi-definite:

$$
\operatorname{det} \mathrm{M}=\prod \lambda^{*} \lambda \geq 0 \text {. }
$$

\footnotetext{
${ }^{\mathrm{b}}$ As before, note that $\gamma_{5}$ anti-commutes with $\mathrm{M}$, so if $M \phi=\lambda \phi$, then $M \gamma_{5} \phi=-\gamma_{5} M \phi=$ $-\lambda \gamma_{5} \phi$.
} 
In what follows we investigate the positivity properties of an effective theory describing only modes near the Fermi surface. To low energy modes, the curvature of the Fermi surface is not evident, and the positivity of the usual Dirac sea determinant is recovered.

A system of degenerate quarks with a net baryon number asymmetry is described by the QCD Lagrangian density with a chemical potential $\mu$,

$$
L_{\mathrm{QCD}}=\bar{\psi} i \not D \psi-\frac{1}{4} F_{\mu \nu}^{a} F^{a \mu \nu}+\mu \bar{\psi} \gamma_{0} \psi,
$$

where the covariant derivative $D_{\mu}=\partial_{\mu}+i A_{\mu}$ and we neglect the quark mass for simplicity.

At high density $\left(\mu \gg \Lambda_{\mathrm{QCD}}\right)$, due to asymptotic freedom the energy spectrum of quarks near the Fermi surface is approximately given by a free Dirac eigenvalue equation,

$$
(\vec{\alpha} \cdot \vec{p}-\mu) \psi_{ \pm}=E_{ \pm} \psi_{ \pm},
$$

where $\vec{\alpha}=\gamma_{0} \vec{\gamma}$ and $\psi_{ \pm}$denote the energy eigenfunctions with eigenvalues $E_{ \pm}=-\mu \pm|\vec{p}|$, respectively. At low energy $E<\mu$, the states $\psi_{+}$near the Fermi surface, $|\vec{p}| \sim \mu$, are easily excited but $\psi_{-}$, which correspond to the states in the Dirac sea, are completely decoupled due to the presence of the energy gap $\mu$ provided by the Fermi sea. Therefore the appropriate degrees of freedom at low energy consist of gluons and $\psi_{+}$only.

Now, we wish to construct an effective theory describing the dynamics of $\psi_{+}$by integrating out modes whose energy is greater than $\mu$. Consider a quark near the Fermi surface, whose momentum is close to $\mu \vec{v}_{F}$. Without loss of generality, we may decompose the momentum of a quark into a Fermi momentum and a residual momentum as

$$
p_{\mu}=\mu v_{\mu}+l_{\mu}
$$

where $v^{\mu}=\left(0, \vec{v}_{F}\right)$. Since the quark energy is given as

$$
E=-\mu+\sqrt{\left(l_{\|}+\mu\right)^{2}+l_{\perp}^{2}},
$$

the residual momentum should satisfy $\left(l_{\|}+\mu\right)^{2}+l_{\perp}^{2} \leq 4 \mu^{2}$ with $\vec{l}_{\|}=\vec{v}_{F} \vec{l} \cdot \vec{v}_{F}$ and $\vec{l}_{\perp}=\vec{l}-\vec{l}_{\|}$.

To describe the small excitations of the quark with Fermi momentum, $\mu \vec{v}_{F}$, we decompose the quark fields as

$$
\psi(x)=e^{i \mu \vec{v}_{F} \cdot \vec{x}}\left[\psi_{+}\left(\vec{v}_{F}, x\right)+\psi_{-}\left(\vec{v}_{F}, x\right)\right],
$$

where

$$
\psi_{ \pm}\left(\vec{v}_{F}, x\right)=P_{ \pm}\left(\vec{v}_{F}\right) e^{-i \mu \vec{v}_{F} \cdot \vec{x}} \psi(x) \quad \text { with } \quad P_{ \pm}\left(\vec{v}_{F}\right) \equiv \frac{1 \pm \vec{\alpha} \cdot \vec{v}_{F}}{2} .
$$


The quark Lagrangian in Eq. (18) then becomes

$$
\begin{aligned}
\bar{\psi}\left(i \not D+\mu \gamma^{0}\right) \psi & =\bar{\psi}_{+}\left(\vec{v}_{F}, x\right) i \gamma_{\|}^{\mu} D_{\mu} \psi_{+}\left(\vec{v}_{F}, x\right) \\
& +\bar{\psi}_{-}\left(\vec{v}_{F}, x\right) \gamma^{0}\left(2 \mu+i \bar{D}_{\|}\right) \psi_{-}\left(\vec{v}_{F}, x\right) \\
& +\left[\bar{\psi}_{-}\left(\vec{v}_{F}, x\right) i \not D_{\perp} \psi_{+}\left(\vec{v}_{F}, x\right)+\text { h.c. }\right]
\end{aligned}
$$

where $\gamma_{\|}^{\mu} \equiv\left(\gamma^{0}, \vec{v}_{F} \vec{v}_{F} \cdot \vec{\gamma}\right), \gamma_{\perp}^{\mu}=\gamma^{\mu}-\gamma_{\|}^{\mu}, \bar{D}_{\|}=\bar{V}^{\mu} D_{\mu}$ with $V^{\mu}=\left(1, \vec{v}_{F}\right)$, $\bar{V}^{\mu}=\left(1,-\vec{v}_{F}\right)$, and $D_{\perp}=\gamma_{\perp}^{\mu} D_{\mu}$.

At low energy, we integrate out all the "fast" modes $\psi_{-}$and derive the low energy effective Lagrangian by matching all the one-light-particle irreducible amplitudes containing gluons and $\psi_{+}$in loop expansion. The effects of fast modes will appear in the quantum corrections to the couplings of low energy interactions. At tree-level, the matching is equivalent to eliminating $\psi_{-}$in terms of equations of motion:

$\psi_{-}\left(\vec{v}_{F}, x\right)=-\frac{i \gamma^{0}}{2 \mu+i D_{\|}} \not D_{\perp} \psi_{+}\left(\vec{v}_{F}, x\right)=-\frac{i \gamma^{0}}{2 \mu} \sum_{n=0}^{\infty}\left(-\frac{i D_{\|}}{2 \mu}\right)^{n} \not D_{\perp} \psi_{+}\left(\vec{v}_{F}, x\right)$.

Therefore, the tree-level Lagrangian for $\psi_{+}$becomes

$$
L_{\text {eff }}^{0}=\bar{\psi}_{+} i \gamma_{\|}^{\mu} D_{\mu} \psi_{+}-\frac{1}{2 \mu} \bar{\psi}_{+} \gamma^{0}\left(D_{\perp}\right)^{2} \psi_{+}+\cdots,
$$

where the ellipsis denotes terms with higher derivatives.

Consider the first term in our effective Lagrangian, which when continued to Euclidean space yields the operator

$$
M_{\mathrm{eft}}=\gamma_{\|}^{E} \cdot D(A)
$$

$M_{\text {eft }}$ is anti-Hermitian and it anti-commutes with $\gamma_{5}$, so it leads to a positive semi-definite determinant. However, note that the Dirac operator is not well defined in the space of $\psi_{+}\left(\vec{v}_{F}, x\right)$ (for fixed $v_{F}$ ), since it maps $\psi_{+}\left(\vec{v}_{F}, x\right)$ into $\psi_{+}\left(-\vec{v}_{F}, x\right)$ :

$$
i \not D_{\|} P_{+} \psi=P_{-} i \not D_{\|} \psi
$$

Since $P_{-}\left(\vec{v}_{F}\right)=P_{+}\left(-\vec{v}_{F}\right), i \not D \psi_{+}\left(\vec{v}_{F}, x\right)$ are $\psi_{+}\left(-\vec{v}_{F}, x\right)$ modes, or fluctuations of a quark with momentum $-\mu \vec{v}_{F}$.

Thus far we have considered the quark velocity as a parameter labelling different sectors of the quark field. This is similar to the approach of heavy quark effective theory $(\mathrm{HQET})^{9}$, in which the velocity of the heavy charm or bottom quark is almost conserved due to the hierarchy of scales between the heavy quark mass and the QCD scale. However, this approach contains 
an ambiguity often referred to as "reparameterization invariance", related to the non-uniqueness of the decomposition (20) of quark momenta into a large and residual component. In the dense QCD case, two $\psi\left(v_{F}, x\right)$ modes whose values of $v_{F}$ are not very different may actually represent the same degrees of freedom of the original quark field. In what follows we give a different formulation which describes all velocity modes of the quark field, and is suitable for defining the quasiparticle determinant.

First, a more precise definition of the breakup of the quark field into Fermi surface modes. Using the momentum operator in a position eigenstate basis: $\vec{p}=-i \vec{\partial}$, we construct the Fermi velocity operator:

$$
\vec{v}=\frac{-i}{\sqrt{-\nabla^{2}}} \frac{\partial}{\partial \vec{x}}
$$

which is Hermitian, and a unit vector.

Using the velocity operator, we define the projection operators $P_{ \pm}$as before and break up the quark field as, $\psi(x)=\psi_{+}(x)+\psi_{-}(x)$, with $\psi_{ \pm}=$ $P_{ \pm} \psi$. By leaving $\vec{v}$ as an operator we can work in coordinate space without introducing the HQET-inspired velocity Fourier transform which introduces $v_{F}$ as a parameter. If we expand the quark field in the eigenstates of the velocity operators, we recover the previous formalism with all Fermi velocities summed up.

The leading low-energy part of the quark action is given by

$$
L_{+}=\bar{\psi} P_{-}(v)\left(i \not \partial-\not A+\mu \gamma_{0}\right) P_{+}(v) \psi .
$$

As before, we define the fields $\psi_{+}$to absorb the large Fermi momentum:

$$
\psi_{+}(x)=e^{-i \mu \vec{x} \cdot \vec{v}} P_{+}(v) \psi(x) .
$$

Let us denote the eigenvalue $v$ obtained by acting on the field $\psi$ (which has momentum of order $\mu$ ) as $v_{l}$ (or $v$ "large"), whereas eigenvalues obtained by acting on the effective field theory modes $\psi_{+}$are denoted $v_{r}$ (or $v$ "residual"). If the original quark mode had momentum $\mathrm{p}$ with $|p|>\mu$ (i.e. was a particle), then $v_{l}$ and $v_{r}$ are parallel, whereas if $|p|<\mu$ (as for a hole) then $v_{r}$ and $v_{l}$ are anti-parallel. In the first case, we have $P_{+}\left(v_{l}\right)=P_{+}\left(v_{r}\right)$ whereas in the second case $P_{+}\left(v_{l}\right)=P_{-}\left(v_{r}\right)$. Thus, the residual modes $\psi_{+}$ can satisfy either of $P_{ \pm}\left(v_{r}\right) \psi_{+}=\psi_{+}$, depending on whether the original $\psi$ mode from which it was derived was a particle or a hole. In fact, $\psi_{+}$modes can also satisfy either of $P_{ \pm}\left(v_{l}\right) \psi_{+}=\psi_{+}$since they can originate from $\psi$ modes with momentum $\sim+\mu v$ as well as $-\mu v$ (both are present in the 
original measure: $D \bar{\psi} D \psi$ ). So, the functional measure for $\psi_{+}$modes contains all possible spinor functions - the only restriction is on the momenta: $\left|l_{0}\right|,|\vec{l}|<\Lambda$, where $\Lambda$ is the cutoff.

In light of the ambiguity between $v_{l}$ and $v_{r}$, the equation $\psi=e^{+i \mu x \cdot v} \psi_{+}$ must be modified to

$$
\psi=\exp (+i \mu x \cdot v \alpha \cdot v) \psi_{+}=\exp \left(+i \mu x \cdot v_{r} \alpha \cdot v_{r}\right) \psi_{+},
$$

where the factor of $\alpha \cdot v_{r}$ corrects the sign in the momentum shift if $v_{r}$ and $v_{l}$ are anti-parallel. In general, any expression with two powers of $v$ is unaffected by this ambiguity. For notational simplicity we define a local operator

$$
X \equiv \mu x \cdot v \alpha \cdot v=\mu \frac{\alpha^{i} x^{j}}{\nabla^{2}} \frac{\partial^{2}}{\partial x^{i} \partial x^{j}} .
$$

Taking this into account, we obtain the following action:

$$
L_{+}=\bar{\psi}_{+} e^{-i X}\left(i \not \partial-\not A+\mu \gamma_{0}\right) e^{+i X} \psi_{+} .
$$

We treat the $\not A$ term separately from $i \not \partial+\mu \gamma_{0}$ since the former does not commute with $\mathrm{X}$, while the latter does. Continuing to Euclidean space, and using the identity $P_{-} \gamma_{\mu} P_{+}=\gamma_{\mu}^{\|} P_{+}$, we obtain

$$
L_{+}=\bar{\psi}_{+} \gamma_{\|}^{\mu}\left(\partial^{\mu}+i A_{+}^{\mu}\right) \psi_{+},
$$

where

$$
A_{+}^{\mu}=e^{-i X} A^{\mu} e^{+i X},
$$

and all $\gamma$ matrices are Euclidean. The term containing $A$ cannot be fully simplified because $[v, A] \neq 0$. Physically, this is because the gauge field carries momentum and can deflect the quark velocity. The redefined $\psi_{+}$ modes are functions only of the residual momenta l, and the exponential factors in the A term reflect the fact that the gluon originally couples to the quark field $\psi$, not the residual mode $\psi_{+}$.

The kinetic term in (35) can be simplified to

$$
\gamma_{\|}^{\mu} \partial^{\mu}=\gamma^{\mu} \partial^{\mu}
$$

since $v \cdot \partial v \cdot \gamma=\partial \cdot \gamma$. The action (35) is the most general dimension 4 term with the rotational, gauge invariance ${ }^{\mathrm{c}}$ and projection properties

If we simultaneously gauge transform $A_{+}$and $\psi_{+}$in (35) the result is invariant. There is a simple relation between the gauge transform of the + fields and that of the original fields: $U_{+}(x)=U(x) e^{i X}$. Of course, the momentum-space support of the + gauge transform must be limited to modes less than the cutoff $\Lambda$. 
appropriate to quark quasiparticles. Therefore, it is a general consequence of any Fermi liquid description of quark-like excitations.

The operator in (35) is anti-Hermitian and leads to a positive, semidefinite determinant since it anti-commutes with $\gamma_{5}$. The corrections given in (26) are all Hermitian, so higher orders in the $1 / \mu$ expansion may reintroduce complexity. The structure of the leading term plus corrections is anti-Hermitian plus Hermitian, just as in the original QCD Dirac Lagrangian with chemical potential.

By integrating out the fast modes, the Euclidean QCD partition function can be rewritten as

$$
Z(\mu)=\int \mathrm{d} A_{+} \operatorname{det} M_{\text {eff }}\left(A_{+}\right) e^{-S_{\text {eff }}\left(A_{+}\right)} .
$$

The leading terms in the effective action for gluons (these terms are generated when we match our effective theory, with energy cutoff $\Lambda$, to QCD) also contribute only real, positive terms to the partition function:

$$
S_{\text {eff }}(A)=\int \mathrm{d}^{4} x_{E}\left(\frac{1}{4} F_{\mu \nu}^{a} F_{\mu \nu}^{a}+\frac{M^{2}}{16 \pi} \sum_{\vec{v}_{F}} A_{\perp \mu}^{a} A_{\perp \mu}^{a}\right) \geq 0,
$$

where $A_{\perp}=A-A_{\|}$and the Debye screening mass is $M=\sqrt{N_{f} /\left(2 \pi^{2}\right)} g_{s} \mu$. Note that Landau damping is due to softer quark modes which have not been integrated out, and therefore do not contribute to matching.

Although the HDET only describes low-energy modes, it still contains Cooper pairing interactions. This is because Cooper pairing, in which the quasiparticles have nearly equal and opposite momenta, is induced by gluonic interactions with small energy and momentum transfer. That is, although a gluon exchange (or other interaction) which causes a large angular deflection of a quasiparticle

$$
|\vec{p}\rangle \rightarrow\left|\vec{p}^{\prime}\right\rangle
$$

must involve a large momentum transfer, and hence is not part of the effective theory, a Cooper pairing interaction

$$
|\vec{p},-\vec{p}\rangle \rightarrow\left|\vec{p}^{\prime},-\vec{p}^{\prime}\right\rangle
$$

only involves a small energy and momentum transfer, even if the angle between $\vec{p}$ and $\vec{p}^{\prime}$ is large. Hence, it is described by the leading order interaction between soft gluons and quarks in the effective theory (35). 


\section{Lattice Simulation}

The goal of this section is to give a method for simulating QCD at finite density. We will consider a chemical potential $\mu$ much larger than $\Lambda_{\mathrm{QCD}}$ throughout, and divide the functional integral over quark excitations into two parts: (I) modes within a shell of width $\Lambda$ of the Fermi surface, and (II) modes which are further than $\Lambda$ from the Fermi surface. We will assume the hierarchy

$$
\mu>>\Lambda>>\Lambda_{\mathrm{QCD}}
$$

The quark determinant in region (I) is well approximated by the determinant of the leading operator in high density effective theory (HDET) as long as the first inequality in (40) is satisfied. As discussed in the previous section, it is positive and real.

Here we will show that the contributions to the effective action for the gauge field from quark modes in region (II) are small and vanish as the $\Lambda$ grows large compared to $\Lambda_{\mathrm{QCD}}$.

First consider the theory in Minkowski space. The Dirac operator is

$$
M=i \not D+\mu \gamma_{0}
$$

and the Dirac equation can be written as

$$
i \partial_{0} \psi=H \psi
$$

with

$$
H=i \alpha \cdot \partial-\mu
$$

a Hermitian operator. The break up into regions (I) and (II) proceeds naturally in terms of energy eigenvalues of $\mathrm{H}$ ( or $l_{0}$ in the HDET notation). The low-lying modes in region (I) are particle states with spatial momenta satisfying $|\vec{p}| \approx \mu$.

The analytic continuation of region (I) to Euclidean space leads to the HDET determinant considered previously.

Modes in region (II) all have large energy eigenvalues, at least as large as $\Lambda$. In considering their effect on physics at the scale $\Lambda_{\mathrm{QCD}}$, we can integrate them out in favor of local operators suppressed by powers of $\Lambda_{\mathrm{QCD}} / \Lambda$.

To make this concrete, consider the effective action for gauge fields with field strengths $F_{\mu \nu}$ of order $\Lambda_{\mathrm{QCD}}$. The quark contribution to this effective action is simply the logarithm of the determinant we wish to compute. It can be expanded diagrammatically in graphs with external gauge field lines connected to a single quark loop. Restricting to region (II), we require that 
the quark modes in the loop have large $H$ eigenvalues. Evaluating such graphs leads only to operators which are local in the external fields $A_{\mu}(x)$.

The resulting renormalizable (dimension 4) operator is the finite density equivalent of $F_{\mu \nu}^{2}$, except that due to the breaking of Lorentz invariance it contains separate time- and space-like components which represent the contribution of high-energy modes to the renormalization of the coupling constant, and Debye screening. These effects do not introduce a complex component when continued to Euclidean space.

Higher dimension operators, which involve additional powers of $F_{\mu \nu}$ or covariant derivatives $D_{\mu}$ are suppressed by the scale $\Lambda$. These are presumably the source of complex terms introduced to the effective action. However, due to the $1 / \Lambda$ suppression they are dominated by the contribution from the low-lying modes in region (I), which is necessarily non-local, but real.

The logarithm of the Euclidean quark determinant will have the form:

$$
\left.\ln \operatorname{det} M \sim O\left(\mu^{4}\right)+(\text { non }- \text { local, real })+O\left(\frac{1}{\Lambda}\right) \text { (local, complex }\right),
$$

where the first term is the (real, constant) free energy of non-interacting quarks, the second term is from the positive determinant in region (I) and the last term is the suppressed, complex contribution from region (II). Only the last two terms depend on the gauge field $A_{\mu}(x)$

On the lattice, one can use the dominant $\operatorname{dependence~of~} \operatorname{det} M$ on the first and second terms to do importance sampling. In order to keep the complex higher dimension operators (last term in (44)) small, it is important that the gauge field strengths are kept smaller than $\Lambda^{2}$. One can impose this condition by using two different lattice spacings, $a_{g}$ for the gluons and $a_{\text {det }}$ for the quarks, with $a_{g}>a_{\text {det }}$. The determinant is calculated on the finer $a_{\text {det }}$ lattice, and is a function of plaquettes which are obtained by interpolation from the plaquettes on the coarser $a_{g}$ lattice. Interpolation can be defined in a natural way, since each lattice link variable $U_{x \mu}$ is an element of the gauge group, and one can connect any two points $g_{1}, g_{2}$ on the group manifold in a linear fashion: $g(t)=g_{1}+t\left(g_{2}-g_{1}\right), 0 \leq t \leq 1$.

\section{Inequalities and Anomaly Matching}

Positivity of the measure allows for rigorous QCD inequalities at asymptotic density. For example, inequalities among masses of bound states can be obtained using bounds on bare quasiparticle propagators. One subtlety that arises is that a quark mass term does not lead to a quasiparticle gap 
(the mass term just shifts the Fermi surface). Hence, for technical reasons the proof of non-breaking of vector symmetries ${ }^{10}$ must be modified. (Naive application of the Vafa-Witten theorem would preclude the breaking of baryon number that is observed in the color-flavor-locked (CFL) phase $\left.{ }^{11}\right)$. A quasiparticle gap can be inserted by hand to regulate the bare propagator, but it will explicitly violate baryon number. However, following the logic of the Vafa-Witten proof, any symmetries which are preserved by the regulator gap cannot be broken spontaneously. One can, for example, still conclude that isospin symmetry is never spontaneously broken (although see below for a related subtlety). In the case of three flavors, one can introduce a regulator $d$ with the color and flavor structure of the CFL gap to show rigorously that none of the symmetries of the CFL phase are broken at asymptotic density. On the other hand, by applying anomaly matching conditions $^{12}$, we can prove that the $S U(3)_{A}$ symmetries are broken. We therefore conclude that the CFL phase is the true ground state for three light flavors at asymptotic density, a result that was first established by explicit calculation ${ }^{13,8,14}$.

To examine the long-distance behavior of the vector current, we note that its correlator in a given background gauge field $A$ can be written as

$$
\left\langle J_{\mu}^{a}\left(\vec{v}_{F}, x\right) J_{\nu}^{b}\left(\vec{v}_{F}, y\right)\right\rangle^{A}=-\operatorname{Tr} \gamma_{\mu} T^{a} S^{A}(x, y ; d) \gamma_{\nu} T^{b} S^{A}(y, x ; d),
$$

where the $S U\left(N_{f}\right)$ flavor current $J_{\mu}^{a}\left(\vec{v}_{F}, x\right)=\bar{\psi}_{+}\left(\vec{v}_{F}, x\right) \gamma_{\mu} T^{a} \psi_{+}\left(\vec{v}_{F}, x\right)$. The propagator with $S U(3)_{V}$-invariant IR regulator $d$ is given as

$$
S^{A}(x, y ; d)=\left\langle x\left|\frac{1}{M}\right| y\right\rangle=\int_{0}^{\infty} \mathrm{d} \tau\left\langle x\left|e^{-i \tau(-i M)}\right| y\right\rangle
$$

where with $D=\partial+i A$

$$
M=\gamma_{0}\left(\begin{array}{cc}
D \cdot V & d \\
d^{\dagger} & D \cdot \bar{V}
\end{array}\right)
$$

Since the eigenvalues of $M$ are bounded from below by $d$, we have

$$
\left|\left\langle x\left|\frac{1}{M}\right| y\right\rangle\right| \leq \int_{R}^{\infty} \mathrm{d} \tau e^{-d \tau} \sqrt{\langle x \mid x\rangle} \sqrt{\langle y \mid y\rangle}=\frac{e^{-d R}}{d} \sqrt{\langle x \mid x\rangle} \sqrt{\langle y \mid y\rangle},
$$

where $R \equiv|x-y|$. The current correlators fall off rapidly as $R \rightarrow \infty$;

$$
\begin{aligned}
& \left|\int \mathrm{d} A_{+} \operatorname{det} M_{\text {eff }}(A) e^{-S_{\text {eff }}}\left\langle J_{\mu}^{A}\left(\vec{v}_{F}, x\right) J_{\nu}^{B}\left(\vec{v}_{F}, y\right)\right\rangle^{A_{+}}\right| \\
& \quad \leq \int_{A_{+}}\left|\left\langle J_{\mu}^{A}\left(\vec{v}_{F}, x\right) J_{\nu}^{B}\left(\vec{v}_{F}, y\right)\right\rangle^{A_{+}}\right| \leq \frac{e^{-2 d R}}{d^{2}} \int_{A_{+}}|\langle x \mid x\rangle||\langle y \mid y\rangle|,
\end{aligned}
$$


where we used the Schwartz inequality in the first inequality, since the measure of the effective theory is now positive, and equation (46) in the second inequality. The IR regulated vector currents do not create massless modes out of the vacuum or Fermi sea, which implies that there is no NambuGoldstone mode in the $S U(3)_{V}$ channel. Therefore, for three massless flavors $S U(3)_{V}$ has to be unbroken as in CFL. The rigorous result provides a non-trivial check on explicit calculations, and applies to any system in which the quasiparticle dynamics have positive measure. The case with non-zero quark masses is complicated, and requires careful consideration of the order of limits ${ }^{6}$.

\section{Conclusion}

The low-energy physics of dense fermionic matter, ranging from quark matter to electronic systems, is controlled by modes near the Fermi surface. An effective Lagrangian describing the low-energy modes can be given in a systematic expansion in powers of the energy scale over the chemical potential. The leading term in this expansion has a simple form, and we have shown that it leads to a real, positive Euclidean path integral measure.

This observation opens the door to importance sampling in Monte Carlo simulations of dense matter systems. The key requirement is that the interactions do not strongly couple the low-energy modes to modes far from the Fermi surface. QCD at high density satisfies this requirement, as do all asymptotically free models. Electronic systems in which the important interactions involve momentum transfer less than the Fermi energy are in this category, although some idealized models such as the Hubbard model are not. We have given some proposals for how the positive effective theory might be simulated numerically. Ultimately, we hope that actual practitioners will develop even more practical methods.

Finally, positivity has analytical applications as well, since it allows the use of rigorous inequalities. In QCD we obtain restrictions on symmetry breaking patterns at high density. Similar restrictions can probably be obtained for electronic systems with suitable interactions.

\section{References}

1. See, for instance, S. Hands, Nucl. Phys. Proc. Suppl. 106, 142 (2002) [arXiv:hep-lat/0109034]; I. M. Barbour, S. E. Morrison, E. G. Klepfish, J. B. Kogut and M. P. Lombardo, Nucl. Phys. Proc. Suppl. 60A, 220 (1998) [arXiv:hep-lat/9705042]; M. G. Alford, Nucl. Phys. Proc. Suppl. 73, 161 (1999) [arXiv:hep-lat/9809166]. 
2. Z. Fodor and S. D. Katz, Phys. Lett. B534, 87 (2002) [arXiv:hep-lat/0104001]; JHEP 0203, 014 (2002) [arXiv:hep-lat/0106002].

3. C. R. Allton et al., Phys. Rev. D66, 074507 (2002) [arXiv:hep-lat/0204010]; P. de Forcrand and O. Philipsen, arXiv:hep-lat/0209084; M. D'Elia and M. P. Lombardo, Phys. Rev. D67, 014505 (2003) [arXiv:hep-lat/0209146].

4. For reviews, see D. K. Hong, Acta Phys. Polon. B32, 1253 (2001) [arXiv:hepph/0101025]; S. D. H. Hsu, [arXiv:hep-ph/0003140], Proceedings of the TMU-Yale Meeting on Dynamics of Gauge Fields, Tokyo, 1999; M. Alford, [arXiv:hep-ph/0102047]; K. Rajagopal and F. Wilczek, [arXiv:hep$\mathrm{ph} / 0011333$.

5. For Japanese Hadronic Facilities, see "Expression of Interest for $\mathrm{Nu}$ clear/Hadron Phys. Exp. at the 50-GeV PS," http://psux1.kek.jp/50GeVPS/EOI-nucl-hadron.pdf; For HADES at GSI, see http://www-hades.gsi.de/.

6. D. K. Hong and S. D. H. Hsu, Phys. Rev. D66, 071501 (2002) [arXiv:hep-ph/0202236]; Phys. Rev. D68, 034011 (2003), [arXiv:hepph/0304156]; [arXiv:hep-lat/0309103], To appear in the proceedings of 21st International Symposium on Lattice Field Theory (LATTICE 2003), Tsukuba, Ibaraki, Japan, 15-19 Jul 2003.

7. D. K. Hong, Phys. Lett. B473, 118 (2000) [hep-ph/9812510].

8. D. K. Hong, Nucl. Phys. B582, 451 (2000) [hep-ph/9905523].

9. N. Isgur and M. B. Wise, Phys. Lett. B232, 113 (1989); ibid. B237, 527 (1990); H. Georgi, Phys. Lett. B240, 447 (1990).

10. C. Vafa and E. Witten, Phys. Rev. Lett. 53, 535 (1984); Nucl. Phys. B234, 173 (1984).

11. M. G. Alford, K. Rajagopal and F. Wilczek, Nucl. Phys. B537, 443 (1999) [arXiv:hep-ph/9804403].

12. S. D. Hsu, F. Sannino and M. Schwetz, Mod. Phys. Lett. A16, 1871 (2001) [arXiv:hep-ph/0006059]; F. Sannino, Phys. Lett. B480 (2000) 280 [arXiv:hepph/0002277]; arXiv:hep-ph/0301035.

13. N. Evans, J. Hormuzdiar, S. D. Hsu and M. Schwetz, Nucl. Phys. B581, 391 (2000) [arXiv:hep-ph/9910313].

14. T. Schafer, Nucl. Phys. B575, 269 (2000) [arXiv:hep-ph/9909574]; I. A. Shovkovy and L. C. Wijewardhana, Phys. Lett. B470, 189 (1999) [arXiv:hep-ph/9910225].

15. P. F. Bedaque and T. Schafer, Nucl. Phys. A697, 802 (2002) [arXiv:hepph/0105150]; D. B. Kaplan and S. Reddy, Phys. Rev. D65, 054042 (2002) [arXiv:hep-ph/0107265].

16. D. K. Hong, T. Lee and D. P. Min, Phys. Lett. B477, 137 (2000) [arXiv:hepph/9912531]; D. K. Hong, Phys. Rev. D62, 091501 (2000) [arXiv:hep$\mathrm{ph} / 0006105]$. 